Pacific Journal of Mathematics

$\boldsymbol{R}$-AUTOMORPHISMS OF $R[t][[X]$ 


\title{
$R$-AUTOMORPHISMS OF $R[t][[X]]$
}

\author{
JoONG-Ho KIM
}

Let $R$ be a commutative ring with identity, $R[t]$ the polynomial ring in an indeterminate $t$ over $R$, and $R[t][[X]]$ the formal power series ring in an indeterminate $X$ over $R[t]$. Let $\alpha=\sum_{i=1}^{\infty} a_{i}(t) X^{i}$ and $\beta=\sum_{i=0}^{\infty} b_{\imath}(t) X^{i}$ be elements of $R[t][[X]]$ where $a_{i}(t)$ and $b_{i}(t)$ are elements of $R[t]$ for each $i$. This paper gives necessary and sufficient conditions in order that there exist an $R$-automorphism of $R[t][[X]]$ mapping $t$ and $X$ onto $\alpha$ and $\beta$ respectively.

Recently O'Malley [3] has considered the $R$-automorphisms of $R[[X]]$ - that is, those automorphisms of $R[[X]]$ which restrict to the identity mapping on $R$. In particular O'Malley has determined necessary and sufficient conditions for existence of an $R$-endomorphism of $R[[X]]$ mapping $X$ onto $\sum_{i=0}^{\infty} \alpha_{i} X^{i}$. In this paper we consider $R$ automorphism $\phi$ of $R[t][[X]]$ such that $\phi(t)$ is not necessarily in $R[t]$. Then we see that any $R$-automorphism of $R[[X]]$ mapping $X$ onto $\beta$ appears as a particular case of an $R$-automorphism of $R[t][[X]]$ mapping $t$ and $X$ onto $\alpha$ and $\beta$ respectively.

All rings considered in this paper are assumed to be commutative and contain an identity element. Throughout this paper, the symbols $\omega$ and $\omega_{0}$ are used to denote the sets of positive and nonnegative integers, respectively.

1. Preliminaries. If $\beta \in R[t][[X]]$ and if $T$ is a subring of $R[t][[X]]$ containing $R$ and $\beta$, then $\left(\beta^{n} T\right)$ will denote the ideal of $T$ generated by $\beta^{n}$, and $(T,(\beta T))$ the topological ring with the $(\beta T)$ adic topology. When $T=R[t][[X]]$, we will simply write $\left(\beta^{n}\right)$ and $(R[t][[X]],(\beta))$ to denote the ideal of $R[t][[X]]$ generated by $\beta^{n}$ and the topological ring $R[t][[X]]$ with the $(\beta)$-adic topology, respectively. It is well known that $(T,(\beta T))$ is a Hausdorff space if and only if $\bigcap_{n s \omega}\left(\beta^{n} T\right)=(0)$, and that if the $(\beta T)$-adic topology is Hausdorff then it is metrizable ([5], p. 51). If $\alpha$ and $\beta$ are elements of $R[t][[X]]$, then $R[\alpha][\beta]$ will be the subring of $R[t][[X]]$ consisting of all forms of $\sum_{i=0}^{n} f_{i}(\alpha) \beta^{i}, n \in \omega_{0}$ where $f_{i}(\alpha)$ is a polynomial in $\alpha$ over $R$ which is obtained by substituting $\alpha$ for $t$ in $f_{i}(t)$. If $f=\sum_{i=0}^{\infty} f_{i}(t) X^{i}$ is a nonzero element of $R[t][[X]$ such that the first nonzero coefficient of $f$ is $f_{k}(t)$, then $f$ has order $k$ and we write $0(f)=k$. If $g(t) \in R[t], \pi_{i}(g(t))$ will denote the coefficient of $t^{i}$ in $g(t)$.

The following theorem was proved by Gilmer [1]. 
THEOREM 1.1. Let $f(t) \in R[t]$ and let $\theta$ be the $R$-endomorphism of $R[t]$ which maps $g(t)$ onto $g(f((t))$ for each $g(t) \in R[t]$. Then $\theta$ is onto (or automorphism) if and only if $\pi_{1}(f(t))$ is a unit of $R$ and $\pi_{i}(f(t))$, for $i \geqq 2$, is nilpotent.

LEMma 1.2. Let $\alpha$ and $\beta$ be elements of $R[t][[X]]$ and suppose that $\bigcap_{n e \omega}\left(\beta^{n}\right)=(0)$. Then there exists an $R$-endomorphism $\phi$ of $R[t][[X]]$ such that $\phi(t)=\alpha$ and $\phi(X)=\beta$, if and only if there exists a subring $T$ of $R[t][[X]]$ containing $R[\alpha][\beta]$ such that $(T,(\beta T))$ is a complete Hausdorff space. Moreover, when such a subring $T$ exists, $\phi$ is unique and $\phi\left(\sum_{i=0}^{\infty} f_{i}(t) X^{i}\right)=\sum_{i=0}^{\infty} f_{i}(\alpha) \beta^{i}$ for each $\left.\sum_{i=0}^{\infty} f_{i}(t) X^{i}\right) \epsilon$ $R[t][[X]]$.

Proof. Let $T$ be a subring of $R[t][[X]]$ containing $R[\alpha][\beta]$ such that $(T,(\beta T))$ is complete. Let $f=\sum_{i=0}^{\infty} f_{i}(t) X^{i} \in R[t][[X]]$. If we consider the sequence $\left\{\sum_{i=0}^{n} f_{i}(\alpha) \beta^{i}\right\}_{n \varepsilon \omega_{0}}$, then it is a Cauchy sequence in the topological ring $R[\alpha][\beta]$ with the $(\beta)$-adic topology and hence a Cauchy sequence in $(T,(\beta T))$. Since $(T,(\beta T))$ is a complete Hausdorff space, $\lim _{n} \sum_{i=0}^{n} f_{i}(\alpha) \beta^{i}$ exists in $T$ and is unique. We define $\phi(f)$ to be $\lim _{n} \sum_{i=0}^{n} f_{i}(\alpha) \beta^{i}$ in $(T,(\beta T))$. Then it is easy to see that $\phi(f)=$ $\phi\left(\sum_{i=0}^{\infty} f_{i}(t) X^{i}\right)=\sum_{i=0}^{\infty} f_{i}(\alpha) \beta^{i}$, and that $\phi(t)=\alpha, \phi(X)=\beta$ and $\phi(r)=$ $r$ for each $r \in R$. Let $f, g \in R[t][[X]]$. Then it is straightforward to show that $\phi(f+g)=\phi(f)+\phi(g)$ and $\phi(f \cdot g)=\phi(f) \cdot \phi(g)$. Therefore, $\phi$ is an $R$-endomorphism of $R[t][[X]]$ such that $\phi(t)=\alpha$ and $\phi(X)=\beta$. Let $\psi$ be any $R$-endomorphism of $R[t][[X]]$ such that $\psi(t)=\alpha$ and $\psi(X)=\beta$. Clearly, $\phi$ and $\psi$ are continuous mappings from a Hausdorff space $(R[t][[X]],(X))$ into a Hausdorff space $(R[t][[X]],(\beta))$, and $\phi$ agrees with $\psi$ on $R[t][X]$. But $R[t][X]$ is a dense subset of $(R[t][[X]]$, $(X))$. Therefore, $\phi=\psi$ and hence $\phi$ is unique.

Conversely suppose that there is an $R$-endomorphism $\dot{\phi}$ of $R[t][[X]]$ such that $\phi(t)=\alpha$ and $\phi(X)=\beta$. Then $\phi$ is a continuous mapping from $(R[t][[X]],(X))$ into $(R[t][[X]],(\beta))$. Let $T=\phi(R[t][[X]])$. Then clearly $T$ is a subring of $R[t][[X]]$ containing $R[\alpha][\beta]$. We show that a Hausdorff space $(T,(\beta T))$ is complete. Let $\left\{f_{n}\right\}_{n s \omega_{0}}$ be a Cauchy sequence of elements in $(T,(\beta T))$. Then there exists a subsequence $\left\{g_{n}\right\}_{n \varepsilon \omega_{0}}$ of $\left\{f_{n}\right\}_{n \varepsilon \omega_{0}}$ such that $g_{n}=\sum_{i=0}^{n} h_{i} \beta^{i}$ for each $n \in \omega_{0}$, where $h_{i} \in$ $T$ for each $i=1, \cdots, n$. Then for each $i \in \omega_{0}$ there exists $p_{i} \in R[[t][[X]]$ such that $\phi\left(p_{i}\right)=h_{i}$, and therefore $\phi\left(\sum_{i=0}^{n} p_{i} X^{i}\right)=\sum_{i=0}^{n} h_{i} \beta^{i}=g_{n}$ for each $n \in \omega_{0}$. Clearly, the sequence $\left\{\sum_{i=0}^{n} p_{i} X^{i}\right\}_{n s \omega_{0}}$ is a Cauchy sequence in the complete Hausdorff space $(R[t][[X]],(X))$, and it converges to $\sum_{i=0}^{\infty} p_{i} X^{i}$. Since $\phi$ is continuous, it follows that $\left\{g_{n}\right\}_{n s \omega_{0}}$ converges to $\phi\left(\sum_{i=0}^{\infty} p_{i} X^{i}\right)$ in $(T,(\beta T))$ and hence $\left\{f_{n}\right\}_{n s \omega_{0}}$ converges to $\phi\left(\sum_{i=0}^{\infty} p_{i} X^{i}\right)$. Thus $(T,(\beta T))$ is a complete metric space. 
THEOREM 1.3. Let $\alpha=\sum_{i=0}^{\infty} a_{i}(t) X^{i}$ and $\beta=\sum_{i=k}^{\infty} b_{i}(t) X^{i}$ be elements of $R[t][[X]], k \geqq 1$. Then there exists a unique $R$-endomorphism $\phi$ of $R[t][[X]]$ such that $\phi(t)=\alpha$ and $\phi(X)=\beta$. Further, $\phi$ is onto if and only if the following conditions are satisfied:

(i) $a_{0}(t)$ is in $\phi\left(R[t][[X]]\right.$ such that $\pi_{1}\left(a_{0}(t)\right)$ is a unit of $R$ and $\pi_{i}\left(a_{0}(t)\right)$, for $i \geqq 2$, is nilpotent;

(ii) $k=1$ and $b_{1}(t)$ is a unit of $R[t]$.

Proof. Since $O(\beta) \geqq 1,(R[t][[X]],(\beta))$ is a complete Hausdorff space. Therefore, by Lemma 1.2 there exists a unique $R$-endomorphism $\phi$ of $R[t][[X]]$ such that $\phi(t)=\alpha$ and $\phi(X)=\beta$. In fact, if $\sum_{i=0}^{\infty} f_{i}(t) X^{i} \epsilon$ $R[t][[X]]$, then $\phi\left(\sum_{i=0}^{\infty} f_{i}(t) X^{i}\right)=\sum_{i=0}^{\infty} f_{i}(\alpha) \beta^{i}$. Suppose that $\phi$ is onto. Then $a_{0}(t) \in \phi(R[t][[X]])$, and for each $f(t) \in R[t]$ there exists $h=$ $\sum_{i=0}^{\infty} h_{i}(t) X^{i} \in R[t][[X]]$ such that $\phi(h)=f(t)$. Since $O(\beta) \geqq 1$ and $\phi(h)=$ $\sum_{i=0}^{\infty} h_{i}(\alpha) \beta^{i}$, the constant term (which is an element of $R[t]$ ) of $\phi(h)$ is $h_{0}\left(a_{0}(t)\right)=f(t)$. Therefore, it follows that the $R$-endomorphism $\theta$ of $R[t]$ which sends $t$ onto $a_{0}(t)$, is onto. Then it follows from Theorem 1.1 that $\pi_{1}\left(a_{0}(t)\right)$ is a unit of $R$ and $\pi_{i}\left(a_{0}(t)\right)$, for $i \geqq 2$, is nilpotent. Since $\phi$ is onto, there exists $g=\sum_{i=0}^{\infty} g_{i}(t) X^{i} \in R[t][[X]]$ such that $\phi(g)=X$. Then the constant term in $\phi(g)=\sum_{i=0}^{\infty} g_{i}(\alpha) \beta^{i}$ considered as a power series in $X$ over $R[t]$, is $g_{0}\left(a_{0}(t)\right)=0$. Since the $R$ endomorphism $\theta$ of $R[t]$ is onto, $\theta$ is an automorphism of $R[t]$ [1]. Thus $g_{0}(t)=0$. Therefore, if $k>1$, then $O(\dot{\phi}(g))>1$ which violates the relation $\phi(g)=X$. Hence $k=1$ and $b_{1}(t) \neq 0$. But the coefficient of $X$ in $\dot{\phi}(g)$ is $g_{1}\left(a_{0}(t)\right) \cdot b_{1}(t)=1$. Therefore, $b_{1}(t)$ is a unit of $R[t]$.

Conversely, suppose that $\phi$ is an $R$-endomorphism of $R[t][[X]]$ mapping $t$ and $X$ onto $\alpha$ and $\beta$ respectively, and that the condition (i) and (ii) are satisfied. Since $a_{0}(t) \in \phi(R[t][[X]])$ and $\phi(t)=\alpha \in \phi(R[t][[X]])$, there exists $h \in R[t][[X]]$ such that $\phi(h)=\alpha-a_{0}(t)$. By Lemma 1.2 there exists a unique $R$-endomorphism $\psi$ of $R[t][[X]]$ such that $\psi(t)=$ $t-h$ and $\psi(X)=X$. Clearly, the condition (i) and Theorem 1.1 show that there exists $d(t) \in R[t]$ such that $d\left(a_{0}(t)\right)=t$. Let $\eta$ be the $R$ endomorphism of $R[t][[X]]$ such that $\eta(t)=d(t)$ and $\eta(X)=X$. Then for each $\sum_{i=0}^{\infty} f_{i}(t) X^{i} \in R[t][[X]]$, it follows that $(\phi \circ \psi \circ \eta)\left(\sum_{i=0}^{\infty} f_{i}(t) X^{i}\right)=$ $(\phi \circ \psi)\left(\sum_{i=0}^{\infty} f_{i}(d(t)) X^{i}\right)=\phi\left(\sum_{i=0}^{\infty} f_{i}(d(t-h)) X^{i}\right)=\sum_{i=0}^{\infty} f_{i}\left(d\left(\alpha-\alpha+a_{0}(t)\right)\right) \beta^{i}=$ $\sum_{i=0}^{\infty} f_{i}\left(d\left(a_{0}(t)\right)\right) \beta^{i}=\sum_{i=0}^{\infty} f_{i}(t) \beta^{i}$. Therefore, $\phi \circ \psi \circ \eta$ is an $R[t]$-endomorphism of $R[t][[X]]$ mapping $X$ onto $\beta$. But $O(\beta)=1$ and $b_{1}(t)$ is a unit of $R$, hence $\phi \circ \psi^{\circ} \circ \eta$ is an $R[t]$-automorphism of $R[t][[X]]$ ([7], p. 137). Therefore $\phi$ is onto and the proof is complete.

Lemma 1.4. Let $\alpha=\sum_{i=0}^{\infty} a_{i}(t) X^{i} \in R[t][[X]]$ such that $a_{0}(t)-$ $\pi_{0}\left(a_{0}(t)\right)$ is regular in $R[t]$, and suppose that $\beta=\sum_{i=k}^{\infty} b_{i}(t) X^{i} \in R[t][[X]]$, $k \geqq 1, b_{k}(t) \neq 0$. Let $\phi$ be the $R$-endomorphism of $R[t][[X]]$ such that $\phi(t)=\alpha$ and $\phi(X)=\beta$. Then if $\phi$ is not one-to-one, $b_{b}(t)$ is a zero 
divisor of $R[t]$.

Proof. Suppose that $\phi$ is not one-to-one. Then there exists $f=$ $\sum_{i=0}^{\infty} f_{i}(t) X^{i} \in R[t][[X]], f \neq 0$ such that $\phi(f)=0$. Since $O(\beta) \geqq 1$, the constant term of $\phi(f)=\sum_{i=0}^{\infty} f_{i}(\alpha) \beta^{i}$ considered as a power series in $X$ over $R[t]$, is $f_{0}\left(a_{0}(t)\right)=0$. But if $a_{0}(t)-\pi_{0}\left(a_{0}(t)\right)$ is a regular element of $R[t]$, it follows that the $R$-endomorphism of $R[t]$ mapping $t$ onto $a_{0}(t)$, is one-to-one ([1], p. 330). Therefore, $f_{0}(t)=0$ and so $O(f) \geqq 1$. If $O(f)=n \geqq 1$, then $f_{n}(t) \neq 0$ and the coefficient of $X^{k n}$ in $\phi(f)$ is $f_{n}\left(a_{0}(t)\right) \cdot\left(b_{k}(t)\right)^{n}=0$. Since $f_{n}(t) \neq 0$ and $a_{0}(t)-\pi_{0}\left(a_{0}(t)\right)$ is regular in $R[t]$, we have that $f_{n}\left(a_{0}(t)\right) \neq 0$. Therefore $\left(b_{k}(t)\right)^{n}$ is a zero divisor of $R[t]$, and hence $b_{k}(t)$ is a zero divisor of $R[t]$.

From Theorem 1.3 and Lemma 1.4 we have the following corollary.

COROLLARY 1.5. Under the hypothesis of Theorem 1.3, $\phi$ is an $R$-automorphism of $R[t][[X]]$ if and only if the following conditions are satisfied:

(i) $a_{0}(t)$ is in $\dot{\phi}\left(R[t][[X]]\right.$ such that $\pi_{1}\left(a_{0}(t)\right)$ is a unit of $R$ and $\pi_{i}\left(a_{0}(t)\right)$, for $i \geqq 2$, nilpotent;

(ii) $k=1$ and $b_{1}(t)$ is a unit of $R[t]$.

2. Main result. In ([4], p. 326) O'Malley and Wood proved the following lemma.

Lemma 2.1. Let $\beta=\sum_{i=0}^{\infty} b_{i} X^{i} \in R[[X]]$. Then there exists an $R$ automorphism $\phi$ of $R[[X]]$ such that $\phi(X)=\beta$ if and only if the following conditions are satisfied:

(i) $(R[[X]],(\beta))$ is a complete Hausdorff space;

(ii) $b_{1}$ is a unit of $R$.

LEMma 2.2. Let $\alpha=\sum_{i=0}^{\infty} a_{i}(t) X^{i}$ and $\beta=\sum_{i=0}^{\infty} b_{i}(t) X^{i}$ be elements of $R[t][[X]]$, and let $\phi$ be an $R$-endomorphism of $R[t][[X]]$ such that $\dot{\phi}(t)=\alpha$ and $\phi(X)=\beta$. Then $b_{0}(t)$ is in the Jacobson radical of $R[t]$ if one of the following conditions is satisfied:

(i) $\pi_{1}\left(a_{0}(t)\right)$ is a unit of $R$, and $\pi_{i}\left(a_{0}(t)\right)$, for $i \geqq 2$, is nilpotent.

(ii) $\phi$ is onto.

Proof. Suppose that the condition (i) is satisfied. Let $f(t) \in R[t]$. Then there exists $g(t) \in R[t]$ such that $g\left(a_{0}(t)\right)=f(t)$. By ([7], p. 131), if 1 denotes a unity of $R[t][[X]]$ then $1+g(t) X$ is a unit of $R[t][[X]]$. Therefore, $\phi(1+g(t) X)=1+g(\alpha) \beta$ is a unit of $R[t][[X]]$, and hence $1+f(t) b_{0}(t)$, the constant term of $1+g(\alpha) \beta$ considered as a power series in $X$ over $R[t]$, is a unit of $R[t]$. But $f(t)$ was an arbitrary element of $R[t]$, so it follows that $b_{0}(t)$ is in the Jacobson radical of $R[t]$. 
Next we suppose that the condition (ii) holds. Then for any $f(t) \in R[t]$, there exists $h \in R[t][[X]]$ such that $\phi(h)=f(t)$. Clearly, $1+h \cdot X$ is a unit of $R[t][[X]]$ and therefore $\phi(1+h \cdot X)=1+f(t) \beta$ is a unit of $R[t][[X]]$. Hence $1+f(t) b_{0}(t)$ is invertible in $R[t]$ for every $f(t) \in R[t]$. So $b_{0}(t)$ is in the Jacobson radical of $R[t]$.

Definition. If $c$ is a nilpotent element of a ring $R$, we define the order of nilpotence of $c$ to be the smallest positive integer $k$ such that $c^{k}=0$.

LEMMA 2.3. Let $\alpha=\sum_{i=0}^{\infty} a_{i}(t) X^{i}$ and $\beta=\sum_{i=0}^{\infty} b_{i}(t) X^{i}$ be elements of $R[t][[X]]$. If $b_{0}(t)$ is in the Jacobson radical of $R[t]$, then the topological ring $(R[t][[X]],(\beta))$ is a Hausdorff and complete space.

Proof. Suppose that $b_{0}(t)$ is in the Jacobson radical of $R[t]$. Then every coefficient of the polynomial $b_{0}(t)$ is nilpotent ([2], p. 152) and hence $b_{0}(t)$ is nilpotent in $R[t]$. Let $n$ be the order of nilpotence of $b_{0}(t)$. Then for each $m \in \omega_{0}, O\left(\beta^{n+m}\right)=O\left(\left(\sum_{i=0}^{\infty} b_{i}(t) X^{i}\right)^{n+m}\right) \geqq m+1$, and therefore it follows that $\bigcap_{n \in \omega}\left(\beta^{n}\right)=(0)$ and that for any sequence $\left\{h_{i}\right\}_{i \in \omega_{0}}$ of elements of $R[t][[X]]$, the sequence $\left\{h_{i} \beta^{i}\right\}_{i \in \omega_{0}}$ is summable in $R[t][[X]]$. Therefore, the topological ring $(R[t][[X]],(\beta))$ is complete and Hausdorff. Moreover, by Lemma 1.2 there is a unique $R$ endomorphism $\phi$ of $R[t][[X]]$ such that $\phi\left(\sum_{i=0}^{\infty} f_{i}(t) X^{i}\right)=\sum_{i=0}^{\infty} f_{i}(\alpha) \beta^{i}$ for each $\sum_{i=0}^{\infty} f_{i}(t) X^{i} \in R[t][[X]]$.

LEMMA 2.4. Let $g(t)=\sum_{i=0}^{n} c_{i} t^{i} \in R[t]$ such that $c_{1}$ is a unit of $R$ and $c_{i}$, for $i \geqq 2$, is nilpotent in $R$. Then for $f(t) \in R[t]$, it follows that $f(t)$ is nilpotent if and only if $f(g(t))$ is nilpotent.

Proof. The lemma is an immediate consequence of Theorem 1.1.

We now prove the most important result of this paper.

THEOREM 2.5. Let $\alpha=\sum_{i=0}^{\infty} a_{i}(t) X^{i}$ and $\beta=\sum_{i=0}^{\infty} b_{i}(t) X^{i}$ be elements of $R[t][[X]]$, and let $\phi$ be an $R$-endomorphism of $R[t][[X]]$ such that $\phi(t)=\alpha$ and $\phi(X)=\beta$. Then $\phi$ is an $R$-automorphism of $R[t][[X]]$ if and only if the following conditions are satisfied:

(i) $a_{0}(t)$ is in $\phi(R[t][[X]])$ such that $\pi_{1}\left(a_{0}(t)\right)$ is a unit of $R$ and $\pi_{i}\left(a_{0}(t)\right)$, for $i \geqq 2$, is nilpotent.

(ii) $b_{1}(t)$ is a unit of $R[t]$.

Proof. $(\rightarrow)$ Suppose that $\phi$ is an $R$-automorphism of $R[t][[X]]$ such that $\phi(t)=\alpha$ and $\phi(X)=\beta$. Then clearly $a_{0}(t) \in \phi(R[t][[X]])$. By Lemma 2.2, $b_{0}(t)$ is in the Jacobson radical of $R[t]$ and hence 
every coefficient of a polynomial $b_{0}(t)$ is nilpotent. Let $P$ be a prime ideal of $R$ distinct from $R$. For each $f(t)=\sum_{i=0}^{n} d_{i} t^{i} \in R[t]$, we define $\bar{f}(t)$ to be $\sum_{i=0}^{n} \bar{d}_{i} t^{i}$ where $\bar{d}_{i}=d_{i}+P \in R / P$ for each $i=1, \cdots, n$, and let $\bar{\alpha}$ and $\bar{\beta}$ be $\sum_{i=0}^{\infty} \bar{a}_{i}(t) X^{i}$ and $\sum_{i=0}^{\infty} \bar{b}_{i}(t) X^{i}$, respectively. Since $\bar{b}_{0}(t)=0$ in $R / P[t]$, from Theorem 1.3 it follows that there exists a unique $R / P$ endomorphism $\phi^{*}$ of $R / P[t][[X]]$ such that $\phi^{*}(t)=\bar{\alpha}$ and $\phi^{*}(X)=\bar{\beta}$. Since $\phi$ is onto, clearly $\phi^{*}$ is onto. Therefore, by Theorem 1.3, it follows that $\pi_{1}\left(\bar{a}_{0}(t)\right)$ is a unit of an integral domain $R / P$ and $\pi_{i}\left(\bar{a}_{0}(t)\right)$, for $i>2$, is 0 in $A / P$, and that $\bar{b}_{1}(t)$ is a unit of $R / P[t]$. Note that $\bar{b}_{1}(t)$ is a unit of $R / P[t]$ if and only if $\pi_{0}\left(\bar{b}_{1}(t)\right)$ is a unit of $R / P$ and $\pi_{i}\left(\bar{b}_{i}(t)\right)$, for $i \geqq 1$, is 0 in $R / P$. Since $P$ was an arbitrary prime ideal of $R$ distinct from $R$, it follows that $\pi_{1}\left(a_{0}(t)\right.$ is a unit of $R$ and $\pi_{i}\left(a_{0}(t)\right)$, for $i \geqq 2$, is nilpotent in $R$, and that $\pi_{0}\left(b_{1}(t)\right)$ is a unit of $R$ and $\pi_{1}\left(b_{1}(t)\right)$, for $i \geqq 1$, is nilpotent. Thus the condition (i) holds and $b_{1}(t)$ is a unit of $R[t]$.

$(\hookleftarrow)$ Suppose that $\phi$ is an $R$-endomorphism of $R[t][[X]]$ such that $\phi(t)=\alpha$ and $\phi(X)=\beta$, and such that the conditions (i) and (ii) are satisfied. Then by Lemma 2.2, $b_{0}(t)$ is in the Jacobson radical of $R[t]$ and therefore every coefficient of a polynomial $b_{0}(t)$ is nilpotent in $R$. We show that $b_{0}(t) \in \phi(R[t][[X]])$. Since $a_{0}(t) \in \phi(R[t][[X]])$, there exists $h \in R[t][[X]]$ such that $\phi(h)=a_{0}(t)$. Let $\theta$ be the $R$-automorphism of $R[t]$ which sends $t$ onto $a_{0}(t)$ [1]. Then there exists $g(t) \in R[t]$ such that $\theta(g(t))=b_{0}(t)$. Clearly $g(h) \in R[t][[X]]$ and $\phi(g(h))=g(\phi(h))=$ $g\left(a_{0}(t)\right)=\theta(g(t))=b_{0}(t)$. Thus $b_{0}(t) \in \phi\left(R[t][[X] 1)\right.$. Let $f=\sum_{i=0}^{\infty} f_{i}(t) X^{i} \in$ $R[t][[x]]$ such that $\phi(f)=b_{0}(t)$. Since $b_{0}(t)$ is nilpotent in $R[t], \bigcap_{n \in \omega}\left(\beta^{n}\right)=$ (0). By Lemma 1.2, $\phi(R[t][[X]])$ is complete and Hausdorff with respect to the $(\beta)$-adic topology, and $\phi\left(\sum_{i=0}^{\infty} f_{i}(t) X^{i}\right)=\sum_{i=0}^{\infty} f_{i}(\alpha) \beta^{i}=b_{0}(t)$. Let $n$ be the order of nilpotence of $b_{0}(t)$. Then the constant term of $\sum_{i=0}^{\infty} f_{i}(\alpha) \beta^{i}$ considered as a power series in $X$ over $R[t]$, is $\sum_{i=0}^{n-1} f_{i}\left(a_{0}(t)\right)\left(b_{0}(t)\right)^{i}$. Then clearly $\sum_{i=0}^{n-1} f_{i}\left(a_{0}(t)\right)\left(b_{0}(t)\right)^{i}=b_{0}(t)$. Therefore, $f_{0}\left(a_{0}(t)\right)=-\sum_{i=1}^{n-1} f_{i}\left(a_{0}(t)\right)\left(b_{0}(t)\right)^{i}+b_{0}(t)$ where $\left(b_{0}(t)\right)^{i}$ is nilpotent in $R[t]$ for each $i=1, \cdots, n-1$. Hence $f_{0}\left(a_{0}(t)\right)$ is nilpotent, and by Lemma 2.4, $f_{0}(t)$ is nilpotent. We show that $f_{1}(t)$ is nilpotent in $R[t]$. Let $f^{\prime}(t)$ be the derivative of $f(t)$ with respect to $t$ for each $f(t) \in R[t]$ ([6], p. 121). Then the coefficient of $X$ in $\sum_{i=0}^{\infty} f_{i}(\alpha) \beta^{i}$ is

$$
\sum_{i=0}^{n-1} f_{i}^{\prime}\left(a_{0}(t)\right) a_{1}(t)\left(b_{0}(t)\right)^{i}+\sum_{i=1}^{n} i \cdot f_{i}\left(a_{0}(t)\right) b_{1}(t)\left(b_{0}(t)\right)^{i-1}=0
$$

and so

(1) $f_{1}\left(a_{0}(t)\right) b_{1}(t)=-\sum_{i=0}^{n-1} f_{i}^{\prime}\left(a_{0}(t)\right) a_{1}(t)\left(b_{0}(t)\right)^{i}-\sum_{i=2}^{n} i f_{i}\left(a_{0}(t)\right) \cdot b_{1}(t)\left(b_{0}(t)\right)^{i-1}$.

Since $f_{0}(t)$ is nilpotent in $R[t], f_{0}(t)$ is in the Jacobson radical of $R[t]$ and therefore each coefficient of $f_{0}(t)$ is nilpotent. Then clearly the 
derivative $f_{0}^{\prime}(t)$ of $f_{0}(t)$ is nilpotent and hence $f_{0}^{\prime}\left(a_{0}(t)\right)$ is nilpotent (by Lemma 2.4). Then from (1) it follows that $f_{1}\left(a_{0}(t)\right) \cdot b_{1}(t)$ is nilpotent. But $b_{1}(t)$ is a unit of $R[t]$, so $f_{1}\left(a_{0}(t)\right)$ is nilpotent and hence $f_{1}(t)$ is nilpotent. Let $\gamma=X-f=X-\sum_{i=0}^{\infty} f_{i}(t) X^{i}$. Then the constant term of $\gamma$ is $f_{0}(t)$. Since $f_{0}(t)$ is nilpotent, $(R[t][[X]],((\gamma))$ is a complete and Hausdorff space (by Lemma 2.3). The coefficient of $X$ in $\gamma$ is $1-f_{1}(t)$ which is a unit of $R[t]$. Therefore, by Lemma 2.1, there exists a unique $R[t]$-automorphism $\psi$ of $R[t][[X]]$ such that $\psi\left(\sum_{i=0}^{\infty} g_{i}(t) X^{i}\right)=$ $\sum_{i=0}^{\infty} g_{i}(t) \gamma^{i}$ for each $\sum_{i=0}^{\infty} g_{i}(t) X^{i} \in R[t][[X]]$. Then $\dot{\phi} \circ \psi$ is an $R$-endomorphism of $R[t][[X]]$, and for each $\sum_{i=0}^{\infty} g_{i}(t) X^{i} \in R[t][[X[]$ we have that

$$
\begin{aligned}
(\phi \circ \psi)\left(\sum_{i=0}^{\infty} g_{i}(t) X^{i}\right) & =\phi\left(\sum_{i=0}^{\infty} g_{i}(t) \gamma^{i}\right)=\phi\left(\sum_{i=0}^{\infty} g_{i}(t)(X-f)^{i}\right) \\
& =\sum_{i=0}^{\infty} g_{i}(\alpha)\left(\beta-b_{0}(t)\right)^{i} .
\end{aligned}
$$

Since $a_{0}(t) \in \phi\left(R[t][[X]]\right.$ and $\psi$ is an automorphism of $R[t][[X]], a_{0}(t)$ is in $(\phi \circ \psi)(R[t][[X]])$. Note that $O\left(\beta-b_{0}(t)\right)=1$ and $b_{1}(t)$ is a unit of $R[t]$. By Corollary 1.5, $\phi \circ \psi$ is an $R$-automorphism of $R[t][[X]]$ which maps $t$ and $X$ onto $\alpha$ and $\beta-b_{0}(t)$, respectively. Hence $\phi$ is an $R$ automorphism of $R[t][[X]]$ and the proof is complete.

Observe that if $\phi$ is any $R$-endomorphism of $R[t][[X]]$ such that $\phi(t)=\alpha$ and $\phi(X)=\beta$, and that is onto, we have the conditions (i) and (ii) stated in Theorem 2.5. Therefore, by the proof of the "if" part of Theorem 2.5, $\phi$ is an automorphism. Thus we have the following.

Corollary 2.6. Let $\alpha=\sum_{i=0}^{\infty} a_{i}(t) X^{i}$ and $\beta=\sum_{i=0}^{\infty} b_{i}(t) X^{i}$ be elements of $R[t][[X]]$, and suppose that $\phi$ is an $R$-endomorphism of $R[t][[X]]$ such that $\phi(t)=\alpha$ and $\phi(X)=\beta$. If $\phi$ is onto, then $\phi$ is an automorphism of $R[t][[X]]$.

From Theorem 2.5 we have the following result.

THEOREM 2.7. Let $\alpha=\sum_{i=0}^{\infty} a_{i}(t) X^{i}$ and $\beta=\sum_{i=0}^{\infty} b_{i}(t) X^{i}$ be elements of $R[t][[X]]$. Then there exists an $R$-automorphism $\phi$ of $R[t][[X]]$ such that $\phi(t)=\alpha$ and $\phi(X)=\beta$, if and only if the following conditions are satisfied:

(i) $b_{0}(t)$ is nilpotent in $R[t]$ and $b_{1}(t)$ is a unit of $R[t]$.

(ii) $a_{0}(t) \in R[\alpha][[\beta]]$ such that $\pi_{1}\left(a_{0}(t)\right)$ is a unit of $R$ and $\pi_{i}\left(a_{0}(t)\right)$, for $i \geqq 2$, is nilpotent.

Proof. Let $\phi$ be an $R$-automorphism of $R[t][[X]]$ such that $\phi(t)=$ $\alpha$ and $\phi(X)=\beta$. Using the same argument as that used in the proof of Theorem 2.5 we see that $b_{0}(t)$ is nilpotent and that $\phi(R[t][[X]])$ is a complete and Hausdorff space with respect to the $(\beta)$-adic topology. 
Thus $R[t][[X]]=\phi(R[t][[X]])=R[\alpha][[\beta]]$. Therefore $a_{0}(t) \in R[\alpha][[\beta]]$. Then by Theorem 2.5, the conditions (i) and (ii) follow immediately.

Conversely we assume the conditions (i) and (ii). Since $b_{0}(t)$ is nilpotent in $R[t],(R[t][[X]],(\beta))$ is a complete and Hausdorff space. Then by Lemma (1.2), there exists a unique $R$-endomorphism of $R[t][[X]]$ such that $\phi(t)=\alpha$ and $\phi(X)=\beta$. Then $\phi(R[t][[X]])$ is a complete and Hausdorff space with the $(\beta)$-adic topology and therefore $\phi(R[t][[X]])=R[\alpha][[\beta]]$. Hence $a_{0}(t) \in \phi(R[t][[X]])$. By Theorem 2.5, $\phi$ is an $R$-automorphism of $R[t][[X]]$.

Corollary 2.8. Let $\beta=\sum_{i=0}^{\infty} b_{i}(t) X^{i} \in R[t][[X]]$, and let $\alpha=g(t)+$ $\sum_{i=1}^{n} a_{i} \beta^{i}$ where $g(t) \in R[t]$ and $a_{i} \in R$ for each $i=1, \cdots, n$. Then there exists an $R$-automorphism $\phi$ of $R[t][[X]]$ such that $\phi(t)=\alpha$ and $\phi(X)=$ $\beta$, if and only if the following conditions are satisfied:

(i) $b_{0}(t)$ is nilpotent in $R[t]$ and $b_{1}(t)$ is a unit of $R[t]$.

(ii) $\pi_{1}(g(t))$ is a unit of $R$ and $\pi_{i}(g(t))$, for $i \geqq 2$, is nilpotent.

Proof. Since $g(t)=\alpha-\sum_{i=1}^{n} a_{i} \beta^{i} \in R[\alpha][[\beta]]$ and $g(t)+\sum_{i=1}^{n} a_{i}\left(b_{0}(t)\right)^{i} \in$ $R[\alpha][[\beta]]$, the corollary is an immediate consequence of Theorem 2.7 and we omit the proof.

REMARK. By Lemma 2.2 and 2.3, we may replace the condition (i) in Theorem 2.7 by the condition " $(R[t][[X]],(\beta))$ is a complete and Hausdorff space and $b_{1}(t)$ is a unit of $R[t] . "$ Then it is easy to see that Lemma 2.1, the main result of O'Malley and Wood [4], appears as a special case of Theorem 2.7.

\section{REFERENCES}

1. R. W. Gilmer, $R$-automorphism of $R[X]$, Proc. London Math. Soc., 18 (1968), 328-336.

2. I. N. Herstein, Noncommutative Rings, Carus Math. Monographs, Math. Assoc. America, (1968).

3. M. J. O'Malley, R-automorphism of $R[[X]]$, Proc. London Math. Soc., 20 (1970), 60-78.

4. M. O'Malley and C. Wood, $R$-endomorphism of $R[[X]]$, J. Algebra, 15 (1970), 314-327.

5. M. Nagata, Local Rings, Interscience, (1962).

6. O. Zariski and P. Samuel, Commutative Alegebra, Vol. I. Van Nostrand, Princeton. N. J. (1958).

7. , Commutative Algebra, Vol. II, Van Nostrand, Princeton, N. J., (1960).

Received March 30, 1971.

East Carolina University 


\section{PACIFIC JOURNAL OF MATHEMATICS}

\section{EDITORS}

H. SAMELSON

Stanford University

Stanford, California 94305

C. R. HobBY

University of Washington

Seattle, Washington 98105
J. DUGUNDJI

Department of Mathematics

University of Southern California

Los Angeles, California 90007

RICHARD ARENS

University of California

Los Angeles, California 90024

\section{ASSOCIATE EDITORS}

E. F. BECKENBACH

B. H. NeumanN

F WoLF

K. YoshidA

\section{SUPPORTING INSTITUTIONS}

UNIVERSITY OF BRITISH COLUMBIA

UNIVERSITY OF SOUTHERN CALIFORNIA

CALIFORNIA INSTITUTE OF TECHNOLOGY

UNIVERSITY OF CALIFORNIA

MONTANA STATE UNIVERSITY

STANFORD UNIVERSITY

UNIVERSITY OF NEVADA

NEW MEXICO STATE UNIVERSITY

OREGON STATE UNIVERSITY

UNIVERSITY OF OREGON

OSAKA UNIVERSITY

UNIVERSITY OF TOKYO

UNIVERSITY OF UTAH

WASHINGTON STATE UNIVERSITY

UNIVERSITY OF WASHINGTON

$\stackrel{*}{*} \stackrel{*}{*} \stackrel{*}{*}{ }^{*}{ }^{*}$ MMEICAN MATHEMATICAL SOCIETY

NAVAL WEAPONS CENTER

Printed in Japan by International Academic Printing Co., Ltd., Tokyo, Japan 


\section{Pacific Journal of Mathematics}

\section{Vol. 42, No. $1 \quad$ January, 1972}

Tage Bai Andersen, On Banach space valued extensions from split faces ........

David Marion Arnold, A duality for quotient divisible abelian groups of finite

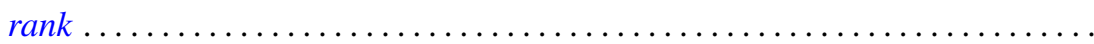

Donald Pollard Ballou, Shock sets for first order nonlinear hyperbolic

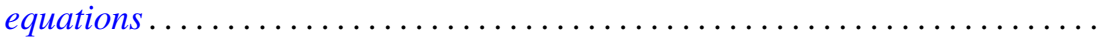

Leon Brown and Lowell J. Hansen, On the range sets of $H^{p}$ functions .........

Alexander Munro Davie and Arne Stray, Interpolation sets for analytic

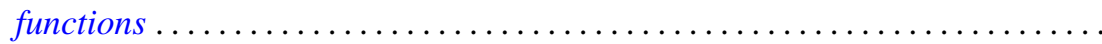

M. G. Deshpande, Structure of right subdirectly irreducible rings. II . . . . . . . . .

Barry J. Gardner, Some closure properties for torsion classes of abelian

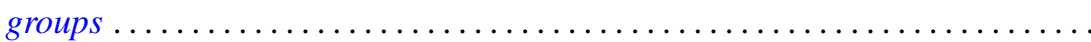

Paul Daniel Hill, Primary groups whose subgroups of smaller cardinality are

direct sums of cyclic groups . . . . . . . . . . . . . . . . . . .

Richard Allan Holzsager, When certain natural maps are equivalences .........

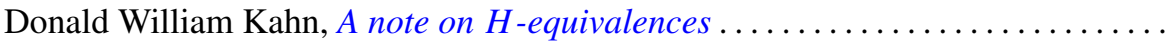

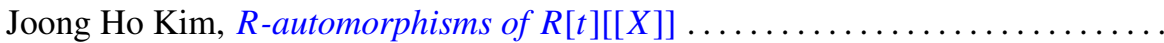

Shin'ichi Kinoshita, On elementary ideals of polyhedra in the 3-sphere.........

Andrew T. Kitchen, Watts cohomology and separability...

Vadim Komkov, A technique for the detection of oscillation of second order

ordinary differential equations .

Charles Philip Lanski and Susan Montgomery, Lie structure of prime rings of characteristic 2

Andrew Lenard, Some remarks on large Toeplitz determinants . .

Kathleen B. Levitz, A characterization of general Z.P.I.-rings. II .

Donald A. Lutz, On the reduction of rank of linear differential systems

David G. Mead, Determinantal ideals, identities, and the Wronskian ...

Arunava Mukherjea, A remark on Tonelli's theorem on integration in product

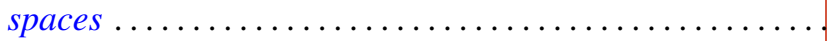

Hyo Chul Myung, A generalization of the prime radical in nonassociative rings.

John Piepenbrink, Rellich densities and an application to unconditionally nonoscillatory elliptic equations.

Michael J. Powers, Lefschetz fixed point theorems for a new class of multi-valued maps .

Aribindi Satyanarayan Rao, On the absolute matrix summability of a Fourier

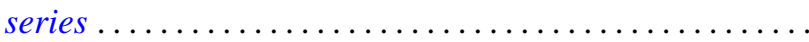

T. S. Ravisankar, On Malcev algebras ......................... 227

William Henry Ruckle, Topologies on sequences spaces . . . . . . . . . . . . . 235

Robert C. Shock, Polynomial rings over finite dimensional rings . . . . . . . . . 251

Richard Tangeman, Strong heredity in radical classes . . . . . . . . . . . . . . 259

B. R. Wenner, Finite-dimensional properties of infinite-dimensional spaces . . . . 267 